Tohoku J. Exp. Med., 2007, 213, 17-23

\title{
Review
}

\section{Problems with Registration-Directed Clinical Trials for Lung Cancer in Japan}

\author{
Ikuo Sekine, ${ }^{1}$ Hiroshi Nokihara, ${ }^{1}$ Noboru Yamamoto, ${ }^{1}$ Hideo Kunitoh, ${ }^{1}$ \\ Yuichiro Ohe, ${ }^{1}$ Nagahiro Saijo ${ }^{2}$ and Tomohide Tamura ${ }^{1}$ \\ ${ }^{1}$ Division of Internal Medicine and Thoracic Oncology, National Cancer Center Hospital, \\ Tokyo, Japan \\ ${ }^{2}$ Division of Internal Medicine, National Cancer Center Hospital East, Kashiwa, Japan
}

Sekine, I., Nokihara, H., Yamamoto, N., Kunitoh, H., Ohe, Y., Saijo, N. and Tamura, T. Problems with Registration-Directed Clinical Trials for Lung Cancer in Japan. Tohoku J. Exp. Med., 2007, 213 (1), 17-23 — New anticancer agents against lung cancer are needed because efficacy of chemotherapy is limited. The long time required, low quality, and considerable costs of registration-directed clinical trials in Japan ("Chiken") have been pointed out. The quality of 24 phase I and 41 phase II trials of an anticancer drug for lung cancer were analyzed according to the approval year of the drug. The human resources and infrastructure to support oncology clinical practice and clinical trials were compared between Japan and the USA. A maximum tolerated dose was not defined in any of seven phase I trials before 1989, and was determined in two of six trials between 1989 and 1996 and in seven of 10 trials thereafter. Before 1989, 29 (20\%) of 142 patients registered in two trials were ineligible, and the number of ineligible patients was not reported in the five trials. Sample size calculations were not performed in any of seven phase II trials before 1989 and were performed in only four of 10 trials between 1989 and 1996 and in all 23 trials conducted thereafter. The shortage of human resources, including medical oncologists, oncology nurse practitioners and clinical research coordinators, is serious and acute. The infrastructure to support clinical trials also remains insufficient in Japan. In conclusion, registration-directed clinical trials of anticancer agents have advanced significantly during last three decades but remain unsatisfactory. The development of infrastructure and human resources is an urgent task to ensure high-quality clinical trials without unnecessary delays. _ clinical trials; medical oncologists; nurse practitioners; lung cancer; anticancer agents

(C) 2007 Tohoku University Medical Press

Lung cancer is one of the most common malignancies and the leading cause of cancerrelated deaths in many countries. In the year 2000 , the annual number of deaths from lung cancer was estimated to be 1.1 million worldwide, and global lung caner incidence is increasing at a rate of $0.5 \%$ per year (Schottenfeld and Searle 2005). About $80 \%$ of patients with lung cancer have already developed distant metastases or pleural effusion, either by the time of the initial

Received June 13, 2007; revision accepted for publication July 11, 2007.

Correspondence: Ikuo Sekine, Division of Internal Medicine and Thoracic Oncology, National Cancer Center

Hospital, Tsukiji 5-1-1, Chuo-ku, Tokyo 104-0045, Japan.

e-mail: isekine@ncc.go.jp 
diagnosis or by the time recurrence is detected after surgery for local disease. These patients can be treated with systemic chemotherapy, but the efficacy of currently available anticancer agents is limited to the extent that patients with advanced disease rarely live long. Therefore, new chemotherapeutic agents continue to be developed against lung cancer (Sekine and Saijo 2000).

The Japanese Pharmaceutical Affairs Law (PAL) was enacted in 1948, and was first amended in 1960 to provide for regulations to ensure the maintenance of the quality, efficacy, and safety of drugs and medical devices, and to promote research and development of these medical and pharmaceutical products. Good Clinical Practice (GCP) was enforced by the Bureau Notification of the Ministry of Health and Welfare of Japan ("Kyokuchou-Tsuuchi”) in 1989 (the former GCP). In 1996, the PAL and its related laws were amended to strengthen GCP (the new GCP), Good Laboratory Practice, Good Post-Marketing Surveillance Practice, and standard compliance reviews, conforming to the International Conference on Harmonization of Technical Requirements for Registration of Pharmaceuticals for Human Use. In contrast to the laws prevailing in the US and EU, marketing approval for anticancer agents in Japan has been granted based on reports of the anti-tumor effects of the new agents in phase II trials (Fujiwara and Kobayashi 2002).

Under this Japanese drug approval system regulated by the PAL, 23 anticancer drugs have been approved for use against lung cancer during the last five decades (Fig. 1). Of these, 9 drugs are original to Japan, some of which are routinely used all over the world. Several problems, however, have been pointed out in registration-directed clinical trials in Japan ("Chiken"), including the long time required, low quality, and considerable cost (The Ministry of Health, Labour and Welfare of Japan 2002; The Ministry of Education, Science and Culture and the Ministry of Health, Labour and Welfare 2003). As a result, Japanese cancer patients must wait for a long time

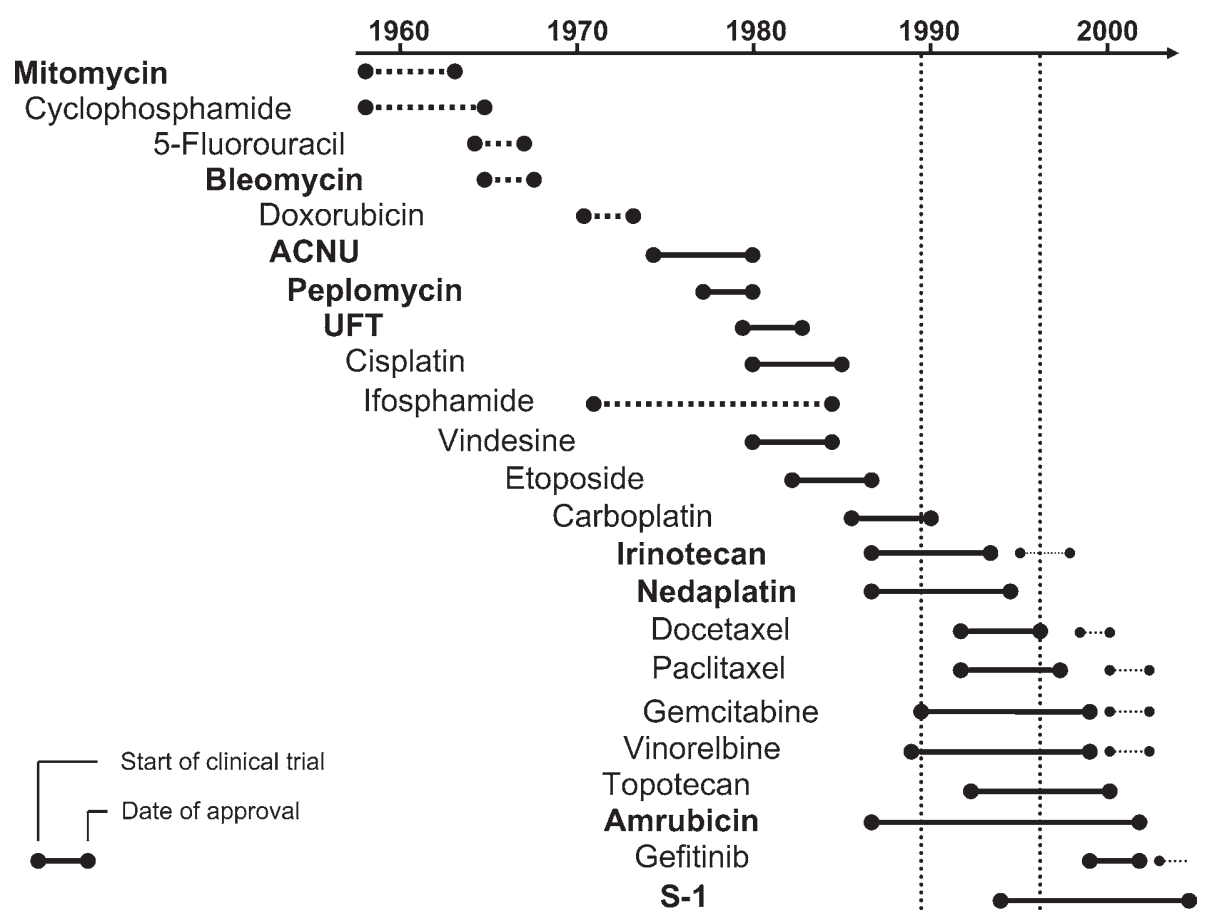

Fig. 1. Anticancer drugs approved for lung cancer in Japan.

Bold: original to Japan. Dotted line: case series studies, solid thick line: investigational new drug phase I-II trials for approval, and dotted thin line: post-marketing sponsored phase III trials. Vertical dotted lines indicate the year when the former and new GCP were issued. 
until they receive new anticancer drugs which have been approved long before in other countries (The Ministry of Health, Labour and Welfare of Japan 2005). We discuss the aspects and issues of registration-directed trials in Japan by reviewing such trials for the 23 anticancer drugs.

\section{Review of registration-directed clinical trials} for the 23 anticancer drugs

A total of 65 phase I and II trials of an anticancer drug for approval were reviewed in terms of definition of eligibility criteria, maximum tolerated dose (MTD), sample size, response criteria, and extramural review for tumor responses. The MTD is the dose associated with seriouis but reversible toxicities in a sizeable proportion of patients and the one that offers the best chance for a favorable therapeutic ratio (Piantadosi 1997). The number of patients accrued in a trial, percentage of ineligible patients, number of participant hospitals in a trial, and the study period defined as the months between the first and last patient accrual were also analyzed. They were obtained from a published paper for 53 trials, from a meeting abstract and in-company resource for one trial, and from in-company resource alone for the remaining 11 trials. The clinical developmental period of an anticancer drug was defined as years between the start month of the first phase I trial and the month of the approval for lung cancer.
These parameters are compared according to the approval year of the drug. We categorized three periods of approval: 1) before 1989, 2) between 1989 and 1996, and 3) between 1997 and 2004, because the former GCP was enforced in 1989, and the new GCP in 1997 (Fujiwara et al. 2002).

Of the 23 anticancer drugs, six drugs whose clinical development started before 1974 were approved on the basis of the clinical experience of the use of the drug without clinical trials (Fig. 1). A total of 24 phase I trials were identified (Table 1). The MTD was not defined in the protocol of any trials before 1989, but was defined in $33 \%$ of trials between 1989 and 1996, and in 70\% of trials after 1996. Instead of the MTD, maximum acceptable dose, defined as the dose associated with grade 2 or severer toxicity in two thirds or more patients, was used in a trial after 1996. About twice more patients were registered in a trial before 1989 than thereafter, but $20 \%$ of the registered patients before 1989 were ineligible. The study period of a phase I trial got longer as the number of participant hospitals decreased, from 7 months and 11 hospitals before 1989 to 13 months and 4 hospitals after 1996, respectively.

In this review, 41 phase II trials for approval were analyzed (Table 2). Calculation of the sample size was not made in any trials before 1989, was seen in $40 \%$ of trials between 1989 and 1996, and in all trials thereafter. Response criteria were

TABLE 1. Investigational new drug phase I trials for approval.

\begin{tabular}{lccc}
\hline & Before 1989 & 1989-1996 & 1997 or thereafter \\
\hline $\begin{array}{l}\text { Total number of trials } \\
\text { Defined, number (\%) of trials }\end{array}$ & 7 & 6 & 11 \\
$\quad$ Eligibility criteria & $4(57)$ & $6(100)$ & $11(100)$ \\
$\quad$ Maximum tolerated dose* & $0(0)$ & $2(33)$ & $7(70)^{\ddagger}$ \\
Results of trials, median (range) & & & \\
$\quad$ Number of patients** & $61(32-170)$ & $24(18-54)$ & $6(9-43)$ \\
$\%$ of ineligible patients & $20(20-21)^{\dagger}$ & $8(0-33)$ & $4(1-17)$ \\
$\quad$ Number of hospitals & $11(1-21)$ & $9(1-18)$ & $13(8-24)$ \\
$\quad$ Study period in months & $7(5-30)$ & $10(5-11)$ & \\
\hline
\end{tabular}

*Statistically significant difference obtained ( $p=0.014$ by the chi-square test); **Statistically significant difference obtained ( $p<0.01$ by the Kruskal Wallis test); ${ }^{\dagger}$ Data were available in 2 trials only; ${ }^{\star}$ Data were available in 10 trials only. 
TABLE 2. Investigational new drug phase II trials for approval.

\begin{tabular}{|c|c|c|c|}
\hline & Before 1989 & 1989-1996 & 1997 or thereafter \\
\hline Total number of trials & 7 & 11 & 23 \\
\hline \multicolumn{4}{|l|}{ Defined, number (\%) of trials } \\
\hline Eligibility criteria & $4(57)$ & $11(100)$ & $23(100)$ \\
\hline Sample size calculation* & $0(0)$ & $4(40)^{\ddagger}$ & $23(100)$ \\
\hline Response criteria & $6(86)$ & $11(100)$ & $23(100)$ \\
\hline Extramural review & $3(43)$ & $9(82)$ & $23(100)$ \\
\hline \multicolumn{4}{|c|}{ Results of trials, median (range) } \\
\hline Number of patients & $71(10-127)$ & $68(18-153)$ & $61(11-102)$ \\
\hline$\%$ of ineligible patients & $18(0-29)^{\dagger}$ & $3(0-22)$ & $3(0-12)$ \\
\hline Number of hospitals & $27(3-103)$ & $17(1-30)$ & $20(5-46)$ \\
\hline Study period in months & $18(12-36)$ & $12(6-34)$ & $26(4-48)^{\S}$ \\
\hline
\end{tabular}

* Statistically significant difference obtained ( $p<0.01$ by the chi-square test); ${ }^{\dagger}$ Data were available in 5 trials only; ${ }^{\star}$ Data were available in 10 trials only; ${ }^{\S}$ Data were available in 22 trials only.

defined in almost all studies, but an extramural review was conducted only after 1989 . The median number of registered patients in a trial was constant through the three periods, but the percentage of ineligible patients was high in trials conducted before 1989. The number of patients in a trial, and the number of hospitals in a trial were similar regardless of the year. The median study period in recent trials was 26 months.

The clinical development period was evaluated in the 23 drugs. Cisplatin was approved for germ cell tumors in 1983 and additionally approved for non-small cell lung cancer (NSCLC) in 1986. S-1 was firstly approved for gastric cancer in 1999, and additionally approved for NSCLC in 2004. The other drugs were approved for lung cancer for the first time. The median (range) clinical development period was 5.2 (3.2-14.5) years before 1989, 6.0 (4.8-9.1) years between 1989 and 1996, and 9.0 (3.9-15.4) years in 1997 or thereafter.

Development and recent problems of phase I and phase II trials in Japan

The concept of the "clinical trial" was not widely followed in Japan until 1974, when a phase I trial of nimustine hydrochloride (ACNU) was launched as one part of the United StatesJapan Cooperation Cancer Research Program on the basis of the agreement between the National Cancer Institute and Japan Society for the Promotion of Science (Sugano 1982; Niitani 1999). Phase I trials before 1989 required the accrual of many patients, because 1) the maximum tolerated dose was not defined, 2) many patients were treated at unnecessary dose levels because the modified Fibonacci dose escalation schedule was not applied, and 3) the percentage of ineligible patients was high. Some of these issues were improved in 1997 or thereafter, but the maximum tolerated dose is still not defined in as many as $40 \%$ of trials. Recently, oncology phase I trials came to be conducted among fewer hospitals than before, as more participants were recruited in each hospital. This facilitated communication among phase I investigators, which is important to complete phase I trials safely.

Phase II trials play the central role in anticancer agent approval in Japan, because the approval can be granted based on the response rate in these trials. The quality of protocols for phase II trials suggested by eligibility criteria, sample size calculation, response criteria, and extramural review has been improved significantly. The study period of phase II trials, however, was and is still too long, as long as 4 years in recent trials. To increase participant hospitals, however, is not necessarily a desirable solution, 
because a certain number of patients per hospital are needed to maintain the quality of trials by training doctors in the application of a new drug. Thus, enhancing patient recruitment in each hospital participating in the trial is the most important consideration.

\section{A high standard of oncology clinical practice as the basis for clinical trials}

Since a high standard of clinical practice is the basis for all clinical trials, the infrastructure for oncological clinical practice should be promptly advanced. The shortage of human resources including medical oncologists and oncology nurse practitioners in Japan is serious and acute. In the United States, medical oncology was established as a separate discipline by the American Board of Internal Medicine in 1971, and approximately 8,000 certified internists as of 2003 have been further certified by the Board in the subspecialty of medical oncology (Holland et al. 2003). In contrast, medical oncology has not been established as an academic unit or a regular university course in many medical schools in Japan. The Japanese Society of Medical Oncology was launched as an association in 1993, and framed the system of cancer medical specialists in 2003. A total of 1,479 doctors were certified as a tentative medical oncology supervisor between 2003 and 2005, and 47 doctors as a medical oncology specialist in 2005 (Table 3) (Japanese Society of Medical Oncology 2005).
To deal with complex cancer care, oncology nurse practitioners in the United States have become an integral part of the multidisciplinary team in the care of patients. As of 2002, more than 19,000 oncology nurse practitioners have been certified by the Oncology Nursing Society in the United States (Rieger 2003). In contrast, the number of oncology nurse practitioners registered in the Japanese Nursing Association was only 44 as of 2005 (Table 3) (Japanese Nursing Association 2005). Introduction of oncology nurse practitioners in clinical practice should lessen the burden on oncologists significantly and help them to have the incentive to take part in registration-directed clinical trials.

\section{The infrastructure and human resources to support clinical trials}

The infrastructure to support in-house clinical trials remains insufficient and even lacking in almost all institutes in Japan, while it has been advanced systematically in the United States. In the 1960s, General Clinical Research Centers were founded with the support of National Institutes of Health in 80 universities and academic institutions to provide the primary resources and optimal environment necessary for investigators to conduct clinical research. They include experienced nursing, laboratory, computer system, and biostatistical staff (Robertson and Tung 2001; General Clinical Research Centers 2005). To carry out a multicenter trial, a central data center

TABLE 3. Medical oncology professionals in Japan and the USA.

\begin{tabular}{lcc}
\hline \multirow{2}{*}{ Professionals } & \multicolumn{2}{c}{$n$ of medical oncology professionals } \\
\cline { 2 - 3 } & Japan & USA \\
\hline Medical oncologists & $47^{1}$ & $8,000^{2}$ \\
Oncology nurse practitioners & $44^{3}$ & $19,000<{ }^{4}$ \\
Clinical research coordinators & $335^{5}$ & $10,723^{6}$ \\
\hline
\end{tabular}

${ }^{1}$ Certified by the Japanese Society of Medical Oncology in 2005.

${ }^{2}$ Certified by the American Board of Internal Medicine as of 2003.

${ }^{3}$ Certified by the Japanese Nursing Association as of 2005.

${ }^{4}$ Certified by the Oncology Nursing Society as of 2002.

${ }^{5}$ Certified by the Japanese Society of Clinical Pharmacology and Therapeutics as of 2005.

${ }^{6}$ Certified by the Association of Clinical Research Professionals as of 2005. 
is needed to deal with the increased administrative difficulties and quality assurance problems associated with this type of trial (Pollock 1994). The quality control and quality assurance system of the Japan Clinical Oncology Group has been significantly developed during the last two decades (Japan Clinical Oncology Group 2005). Using Internet resources may facilitate developing national and regional networks for clinical trials by reducing the burden associated with the extensive research time and considerable cost of all these processes (Paul et al. 2005).

The new GCP demands more of the clinical researchers in time, resources and money to enhance the science, credibility, and ethics of clinical trials for approval (Sweatman 2003). The clinical research coordinator (CRC) plays a key role in the clinical trial process by supporting investigators. The CRCs are involved in every aspect of registration-directed clinical trials, including protocol development, checking eligibility criteria, informed consent, organizing study schedules, checking clinical tests, filling in case report forms, and providing support for monitoring and auditing the trials (Rico-Villademoros et al. 2004; Sakamoto 2004). Association of Clinical Research Professionals in the USA has offered the CRC certification since 1992, and there are 10,723 CRCs to date (Association of Clinical Research Professionals 2006). The Japanese Society of Clinical Pharmacology and Therapeutics launched the certified CRC system in 2003, and there were 335 certified CRCs as of 2005 (Table 3) (The Japanese Society of Clinical Pharmacology and Therapeutics 2005).

In conclusion, clinical trials of anticancer agents for approval have been developing significantly, but still remain at an unsatisfactory level. Development of the infrastructure and human resources for clinical trials is an urgent task to complete good quality clinical trials for approval without delay.

\section{Acknowledgments}

We thank Yuko Yabe and Mika Nagai for kindly preparing this manuscript.

\section{References}

Association of Clinical Research Professionals (2006) [Cited 6 April 2006.] Available, http://www.acrpnet.org/

Fujiwara, Y. \& Kobayashi, K. (2002) Oncology drug clinical development and approval in Japan: the role of the pharmaceuticals and medical devices evaluation center (PMDEC). Crit. Rev. Oncol. Hematol., 42, 145-155.

General Clinical Research Centers (2005) [Cited 4 Aug 2005.] Available, http://www.ncrr.nih.gov/clinical/cr_gcrc.asp2005

Holland, J., Frei, E. \& Kufe, D.W. \& Bast, R.C., Jr. (2003) Principles of medical oncology. In: Cancer Medicine, edited by D.W. Kufe, R.E. Pollock, R.R. Weichselbaum, R.C. Bast, Jr., T.S. Gansler, J.F. Holland \& E. Frei, III., 6th ed., BC Decker Inc., Hamilton, 637-644.

Japan Clinical Oncology Group (2005) [Cited 4 Aug 2005.] Available, http://www.jcog.jp/2005

Japanese Nursing Association (2005) [Cited 4 Aug 2005.] Available, http://www.nurse.or.jp/2005 (in Japanese)

Japanese Society of Medical Oncology (2005) [Cited 4 Aug 2005.] Available, http://jsmo.umin.jp/2005. (in Japanese)

Niitani, H. (1999) Short history of the clinical developments in lung cancer treatment. Gan To Kagaku Ryoho, 26, Suppl. 1, 110-117. (in Japanese)

Paul, J., Seib, R. \& Prescott, T. (2005) The Internet and clinical trials: background, online resources, examples and issues. J. Med. Internet Res., 7, e5.

Piantadosi, S. (1997) Clinical trials as experimental designs. In: Clinical Trials. A Methodological Perspective, edited by S. Piantadosi, John Wiley \& Sons, Inc., New York, pp. 61-105.

Pollock, B.H. (1994) Quality assurance for interventions in clinical trials. Multicenter data monitoring, data management, and analysis. Cancer, 74, 2647-2652.

Rico-Villademoros, F., Hernando, T., Sanz, J.L., Lopez-Alonso, A., Salamanca, O., Camps, C. \& Rosell, R. (2004) The role of the clinical research coordinator - data managerin oncology clinical trials. BMC Med. Res. Methodol., 4, 6.

Rieger, P. \& Yarbro, C. (2003) Principles of Oncology Nursing. In: Cancer Medicine, edited by D.W. Kufe, R.E. Pollock, R.R. Weichselbaum, R.C. Bast, Jr., T.S. Gansler, J.F. Holland, \& E. Frei, III, 6th ed., BC Decker Inc., Hamilton, pp. 1055-1062.

Robertson, D. \& Tung, C.S. (2001) Linking molecular and bedside research: designing a clinical research infrastructure. $J$. Mol. Med., 79, 686-694.

Sakamoto, T. (2004) Chemotherapy and clinical research coordinator. Gan To Kagaku Ryoho, 31, 22-26. (in Japanese)

Schottenfeld, D. \& Searle, J.G. (2005) The etiology and epidemiology of lung cancer. In: Lung Cancer: Principles and Practice, edited by H.I. Pass, D.P. Carbone, J.D. Minna, D.H. Johnson \& A.T. Turrisi, III., 3rd ed., Lippincott Williams \& Wilkins, Philadelphia, pp. 3-24.

Sekine, I. \& Saijo, N. (2000) Novel combination chemotherapy in the treatment of non-small cell lung cancer. Expert Opin. Pharmacother., 1, 1131-1161.

Sugano, H. (1982) The United States-Japan Cooperation Cancer Research Program. Taisha, 19, 1225-1228. (in Japanese)

Sweatman, J. (2003) Good clinical practice: a nuisance, a help or a necessity for clinical pharmacology? Br. J. Clin. Pharmacol., 55, 1-5.

The Japanese Society of Clinical Pharmacology and Therapeutics (2005) [Cited 4 Aug 2005.] Available, http://www.jade. dti.ne.jp/ clinphar/2005 (in Japanese) 
The Ministry of Education, Science and Culture and the Ministry of Health, Labour and Welfare (2003) The three-year project for activating clinical trials for approval in Japan. [Cited 4 Aug 2005.] Available, http://www.mhlw.go.jp/ topics/bukyoku/isei/chiken/kasseika.html2003 (in Japanese)

The Ministry of Health, Labour and Welfare of Japan (2002) A vision of the pharmaceutical industry in Japan. [Cited 4 Aug 2005.] Available, http://www.mhlw.go.jp/shingi/2002/ 08/s0830-1.html2002 (in Japanese)

The Ministry of Health, Labour and Welfare of Japan (2005) Council for the use of unapproved drugs. [Cited 4 Aug 2005.] Available, http://www.mhlw.go.jp/shingi/2005/01/ s0124-9.html2005 (in Japanese) 